\title{
IMPACTO FINANCEIRO DA NÃO-INTEGRAÇÃO DE ESTRADAS EM MODELOS DE AGENDAMENTO TÁTICO DE COLHEITA
}

\author{
FINANCIAL IMPACT OF ROADS NON-INTEGRATION IN TACTICAL HARVEST SCHEDULING
}

\author{
Linamara Smaniotto Ferrari ${ }^{1}$, Julio Eduardo Arce ${ }^{2}$, Allan Libanio Pelissari ${ }^{3}$, \\ Cassius Tadeu Scarpin ${ }^{4}$, Renata Naoko Correa
}
1, 2, 3, 4 Universidade Federal do Paraná, Curitiba, Paraná, Brasil - linamara ferrari@hotmail.com, jarce@ufpr.br, allanpelissari@gmail.com,\&cassiusts@gmail.com

${ }^{5}$ Instituto Federal Catarinense, Santa Rosa do Sul, Santa Catarina, Brasil-naokorenata@gmail.com

\section{RESUMO}

No planejamento tático florestal, as decisões relacionadas ao agendamento da colheita são altamente interligadas às decisões do planejamento de estradas. Porém, devido à complexidade de integrar problemas de colheita florestal e estradas em um mesmo modelo, a abordagem desse tipo de problema tem sido tradicionalmente realizada em duas etapas sequencialmente vinculadas. Por esse motivo, este estudo tem como objetivo comparar as consequências financeiras da não integração do planejamento das estradas em modelos de agendamento tático da colheita florestal através da aplicação da Programação Linear Inteira Mista (PLIM). O modelo PLIM foi implementado em uma floresta pertencente a uma indústria brasileira de base florestal. Com base em dados geoespaciais, foi aplicado o algoritmo de Dijkstra para gerar os caminhos mais curtos entre cada talhão florestal e o destino final (fábrica). No entanto, tendo em vista a seleção prioritária de determinados tipos de estradas, o algoritmo de Dijkstra foi adaptado para penalizar de maneira mais intensa os trechos de estrada com menor capacidade de tráfego de caminhões e maquinários, como por exemplo estradas estreitas e de terra. Os cenários testados resultaram em soluções inteira ótima em menos de um minuto de processamento computacional. Além disso, a aplicação de um modelo integrado de agendamento da colheita florestal com o planejamento das estradas elevou em 0,6\% a receita líquida total do planejamento florestal tático, bem como reduziu em $21 \%$ a quantidade de estradas necessárias às operações de colheita da madeira.

PALAVRAS-CHAVE: Otimização, Planejamento integrado, Programação linear inteira mista.

\section{ABSTRACT}

In tactical forest planning, harvest scheduling decisions are highly interconnected with road planning decisions. However, due to the complexity of integrating forest harvesting and roads problems in the same model, the approach to this type of problem has traditionally been carried out in two sequentially linked steps. For this reason, this study aims to compare the financial consequences of not integrating road planning into models of tactical scheduling of forest harvest through the application of Mixed Linear Integer Programming (PLIM). The MILP model was implemented on a plantation owned by a Brazilian forest-based company. Based on geospatial data, the Dijkstra algorithm was applied to generate the shortest paths between each forest stand and the destination (company). However, Dijkstra's algorithm was adapted to penalize more intensely the road sections with less traffic capacity of trucks and machinery, such as narrow and dirt roads. The tested scenarios obtained optimal integer solutions in less than a minute. In addition, the integrated forest harvest scheduling model with road planning increased the total net revenue from tactical forest planning by $0.6 \%$, as well as reduced the number of roads needed for harvesting operations by $21 \%$.

KEYWORDS: Optimization, Integrated planning, Mixed-integer linear programming. 


\section{INTRODUÇÃO}

O planejamento hierárquico aplicado para a resolução de problemas de programação de colheita de madeira tem sido tradicionalmente utilizado por gestores florestais como auxílio à tomada de decisão (BELAVENUTTI et al., 2019). Essa abordagem decompõe o problema em decisões estratégicas, táticas e operacionais, de maneira que os objetivos do mais alto nível sejam mantidos nos planejamentos subsequentes, variando apenas em uma escala de informações mais detalhadas e de menor período de tempo. Tipicamente, o planejamento tático de colheita florestal aborda dados espaciais que determinam onde e quando os talhões devem ser cortados, de modo a atender às demandas temporais de madeira (RÖNNQVIST et al., 2015). Além disso, o planejamento tático pode incluir decisões de construção e manutenção de estradas (MCDILL, 2014), uma vez que elas garantem o acesso às áreas de corte (RÖNNQVIST et al., 2015).

No planejamento tático, as decisões relacionadas à seleção de estradas florestais limitadas a um orçamento anual são fatores interligados às decisões de quais áreas de colheita devem ser selecionadas em cada ano (MOBTAKER et al., 2020). Isso porque os custos associados a colheita e estradas são elevados, e podem impactar na viablidade de um plano de manejo (D'AMOURS et al., 2010). Portanto, torna-se pertinente integrar decisões de agendamento da colheita ao planejamento de estradas em um único modelo conforme inicialmente apresentadas por Weintraub \& Navon (1976) e Kirby et al. (1986).

No entanto, quando utilizadas variáveis binárias (1-0) para representar áreas de corte e estradas em um planejamento integrado, a solução do problema pode se tornar complexa em termos de tempo polinomial não determinístico (NP-Hard), dado ao grande tamanho e número de variáveis do problema (GUIGNARD; RYU; SPIELBERG, 1998; NADERIALIZADEH et al., 2020). Devido a complexidade de problemas que integram a colheita florestal com a rede de estradas faz com que ainda seja comum a abordagem desse tipo de problema em duas etapas sequencialmente vinculadas (BORGES et al., 2014). Isto é, na primeira etapa o modelo de agendamento da colheita identifica as áreas que serão colhidas, para que então, sejam definidas as estradas de acessos às áreas de corte (GUIGNARD et al., 1998, BORGES et al., 2014).

A tomada de decisão em duas etapas sequencialmente vinculadas pode levar a obtenção de valores sub-ótimos de um problema (GUIGNARD et al., 1998). Dessa maneira, este estudo tem como objetivo comparar as consequências financeiras da não integração do planejamento das estradas em modelos de agendamento tático da colheita florestal através da aplicação de modelos de Programação Linear Inteira Mista (PLIM). Para isso, a hipótese formulada neste estudo baseia-se que o processamento em duas etapas sequencialmente vinculadas do agendamento da colheita e de estradas influencia em maiores custos operacionais que quando trabalhadas de maneira integrada.

\section{MATERIAL E MÉTODOS}

\section{Definição do conjunto de estradas candidatas a serem consideradas no modelo matemático}

A definição das redes de estradas florestais utilizadas como dados de entrada para um modelo matemático devem garantir o atendimento de requisitos técnicos mínimos para seleção de segmentos de estradas, bem como assegurar a acessibilidade à todas unidades de manejo da floresta (BORGES et al., 2014). Dessa maneira, a acessibilidade às unidades de manejo deste estudo foi estabelecida conforme a estrada mais próxima ao centroide de cada talhão, as quais foram definidas a partir do uso de dados geoespaciais (shapefile) executados no software QGIS 3.14.16. Na sequência, o algoritmo de Dijkstra (DIJKSTRA, 1959) foi aplicado para gerar os caminhos mais curtos de escoamento da madeira entre cada talhão florestal até o destino final (fábrica).

No entanto, tendo em vista a seleção prioritária de determinados tipos de estradas, o algoritmo de Dijkstra foi adaptado para penalizar trechos de estrada menos consolidados. Ou seja, estradas estreitas e de terra, como estradas do tipo secundária, receberam penalizações mais expressivas que em relação a estradas mais largas com cascalho ou asfalto, visando minimizar o uso deste tipo de estrada que não permitem intenso tráfego de caminhões e maquinários. Assim, diferentes pesos foram estabelecidos conforme o tipo de estrada e multiplicados pela distância de cada segmento, de modo que o algoritmo priorizasse a ordem: asfalto, estrada municipal, estrada principal e estrada secundária.

Estabelecidos os caminhos mais curtos de estradas candidatas a serem consideradas no modelo matemático, a rede de estradas foi modelada como um grafo, em que cada segmento de estrada foi representado por um arco direcionado conectado por nós fonte $m$ e nós coletores $n$. A partir da associação de cada talhão florestal com os nós conectados à rede de estrada, permitiu-se conectar todo o volume produzido na floresta até o nó final (fábrica) em cada ano $j$ (BORGES et al., 2014).

O algoritmo de Dijkstra foi implementado em 
linguagem Python, utilizando a biblioteca NetworkX e a função "dijkstra_path" para a definição do caminho mais curto.

\section{Modelo matemático}

A formulação do problema de planejamento integrado foi baseada em uma abordagem PLIM, conforme apresentada na sequência:

Função objetivo

$$
\max \sum_{i=1}^{|H|} \sum_{j=1}^{|J|}\left(l_{i j} \cdot x_{i j}\right)-\sum_{(m, n) \in Y} \sum_{j=1}^{|J|}\left(c_{m n j} \cdot z_{m n j}\right)
$$

Restrições

$$
\begin{array}{lc}
\sum_{j=1}^{|J|} x_{i j} \leq 1 & \forall i \in H \\
\sum_{i=1}^{|H|}\left(v_{i j p} \cdot x_{i j}\right) \geq \tau_{p} & \forall j \in J, \forall p=1 \\
\sum_{i=1}^{|H|}\left(\omega_{i j} \cdot x_{i j}\right) \leq \sigma & \forall j \in J \\
\left(n_{i}+j-1\right) \cdot x_{i j} \geq \eta \cdot x_{i j} & \forall i \in I, \forall j \in J \\
\left(n_{i}+j-1\right) \cdot x_{i j} \leq \beta \cdot x_{i j} & \forall i \in I, \forall j \in J \\
f_{m n j}=\sum_{i=1}^{|H|}\left(\omega_{i j} \cdot x_{i j}\right) & \forall(m, n) \in N_{\bar{c}}, \\
\sum_{i \in H_{n}}\left(\omega_{i j} \cdot x_{i j}\right)+\sum_{(m, n) \epsilon Y} f_{m n j}-\quad \sum_{(n, o) \epsilon Y} f_{n o j}=0 \\
f_{m n j}+f_{n m j} \leq M . z_{m n j} & \forall n \in N \backslash\{\bar{c}\}, \forall j \in J \\
x_{i j}, z_{m n j} \in\{0,1\} \text { e } f_{m n j} \geq 0 & \forall(m, n) \in Y, \forall j \in J
\end{array}
$$

Onde: $H$ representa o conjunto dos talhões florestais, e $|H|$ a cardinalidade do respectivo conjunto; J é o conjunto de períodos no horizonte de planejamento; $P$ representa o conjunto de sortimentos gerados pela floresta; $N$ corresponde ao conjunto de nós presente na rede de estradas; $Y$ representa um conjunto dos potenciais segmentos de estrada; $N_{\bar{c}}$ corresponde ao conjunto de nós que estão conectados ao destino final $\bar{c} ; x_{i j}$ é uma variável binária (0 ou 1) que indica se o talhão $i$ é agendado para colheita no período $j ; z_{m n j}$ é uma variável binária que representa o uso ou não do segmento de estrada entre o nó fonte $m$ e $n$ nó coletor $n$ no período $j ; f_{m n j}$ representa o fluxo de volume a ser transportado ao longo do segmento de estrada entre os nós $m$ e $n$ no período $p ; l_{i j}$ representa o valor presente líquido $(R \$)$ relacionado ao talhão $i$ no período $j ; \quad v_{i j p}$ corresponde ao volume produzido pelo sortimento de madeira $p$ caso o talhão $i$ seja selecionado para corte no período $j ; \omega_{i j}$ representa o volume total produzido pelo talhão $i$ no período $j ; \tau_{p}$ representa a demanda mínima de produção anual $\left(\mathrm{m}^{3}\right)$ do sortimento $p ; \sigma$ corresponde a capacidade máxima de produção anual $\left(\mathrm{m}^{3}\right) ; n_{i}$ representa a idade do talhão $i$ no primeiro período do horizonte de planejamento; $\eta$ representa a idade mínima de corte; $\beta$ corresponde a idade máxima de corte; $c_{m n j}$ representa o custo corrente $(\mathrm{R} \$$ ) de manutenção do segmento de estrada entre os nós $m$ e $n$ no período $p$; e $M$ representa um número arbitrariamente grande.

A função objetivo (1) tem a finalidade de maximizar os valores presentes das receitas de colheita florestal menos os custos descontados de manutenção de estradas. A função objetivo é composta por dois somatórios. O primeiro representa o valor total da receita menos os custos operacionais relacionados à colheita florestal. Em contrapartida, o segundo somatório expressa o custo presente total de manutenção das estradas.

A restrição (2) garante que cada talhão seja designado para corte apenas uma vez durante o horizonte de planejamento. A restrição (3) assegura a produção mínima anual do sortimento de madeira para processo. A restrição (4) estabelece a capacidade máxima de produção em cada período $j$. As inequações (5) e (6) estabelecem que os talhões só podem ser colhidos dentro do intervalo de idade mínima e máxima de corte estabelecido. O conjunto de restrições de (7) à (9) estão relacionados às estradas. A equação (7) garante que o nó conectado ao destino final da rede de estrada (fábrica) receba o volume total produzido em cada ano do planejamento. O conjunto de restrições (8) garante que o volume de madeira proveniente de cada talhão florestal associado aos nós conectados à rede de estradas $\left(f_{m n j}, f_{n o j}\right)$, com exceção do nó final $\bar{c}$, sejam igualados ao volume total que deixa o nó, em um dado período $j$ (BORGES et al., 2014). O conjunto de restrições (10) garantem que, caso haja fluxo de madeira no segmento de estrada $m$ e $n$ no período $j$, os custos de manutenção sejam aplicados apenas uma vez em cada período.

Os cenários foram escritos em linguagem Python e resolvidos via Gurobi 9.0.3, em um computador Intel ${ }^{\circledR}$ Core $^{\text {TM }}$ i7-8550U $2.00 \mathrm{GHz}$ de processador e $8 \mathrm{~GB}$ de RAM. Para a resolução de cada cenário testado, foi definido um tempo de processamento computacional máximo de três horas.

\section{Estudo de caso}

O estudo de caso foi fundamentado em dados reais 
pertencentes a uma empresa de base florestal localizada na região Sul do Brasil. Conforme a classificação de Köppen (ALVARES et al., 2013), o clima da região de estudo é caracterizado como temperado ( $\mathrm{Cfb}$ ), com temperatura média abaixo de $18^{\circ} \mathrm{C}$ no mês mais frio, com ocorrência de geadas. A área total da floresta testada foi composta por 3.427,06 ha de Pinus taeda L., subdividida em 805 talhões com área média de 4,2 ha. O horizonte de planejamento foi definido para 5 anos, dividido em períodos anuais. A idade dos talhões florestais variou de 9 a 18 anos, com atual distribuição desigual de classes de idade entre talhões (Figura 1). A floresta é manejada para produzir madeira para processo como principal produto florestal para abastecimento de uma das fábricas da empresa. Além da madeira para processo, essa floresta produz madeira para serraria, que é considerado como volume excedente e comercializado no mercado local. As estimativas de crescimento do povoamento foram definidas e fornecidas pela empresa.

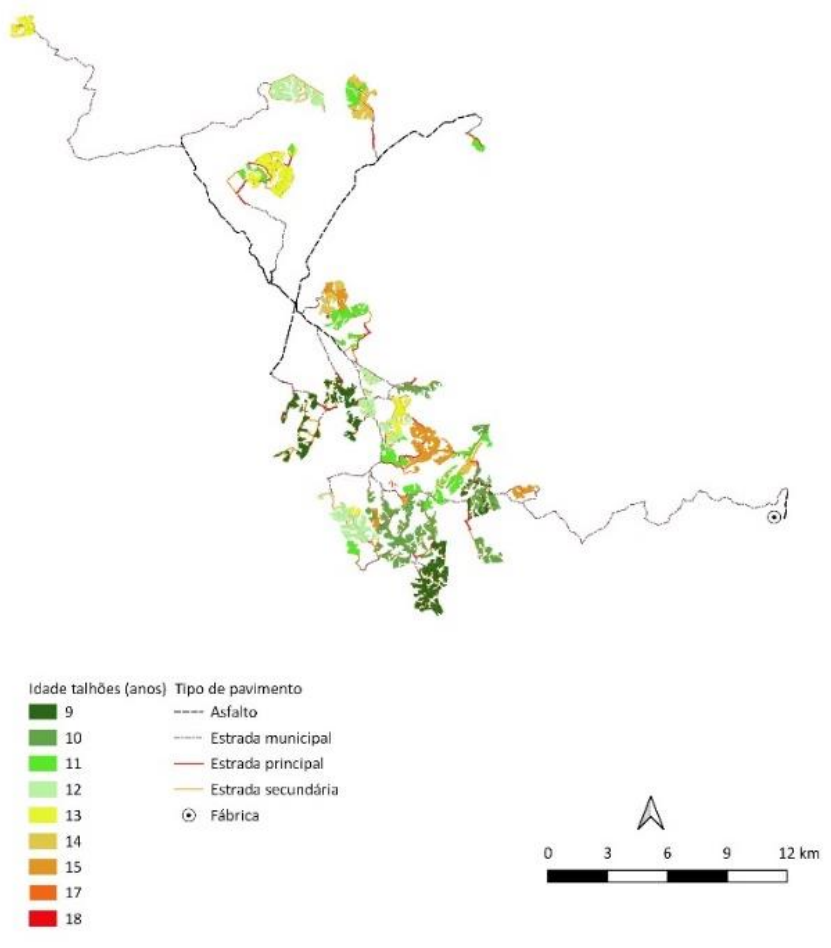

Figura 1. Distribuição espacial dos talhões florestais de acordo com a faixa etária.

Neste estudo foram implementados dois cenários com o objetivo de comparar as consequências financeiras da não integração do planejamento das estradas em modelos de agendamento tático da colheita florestal. O primeiro cenário considerou o planejamento de colheita florestal e das estradas florestais em duas etapas sequencialmente vinculadas, enquanto o segundo considerou o planejamento da colheita florestal integrado ao de estradas.

Ambos os cenários utilizaram a mesma função objetivo (1) e as restrições de (2) à (10), os quais se diferenciaram quanto a não aplicação dos custos de estrada no Cenário 1 e a aplicação dos custos de estradas no Cenário 2. Essa metodologia permite que o Cenário 1 selecione as áreas de corte sem sofrer influência dos custos de estradas. Além de que, permite que os segmentos de estrada selecionados após a otimização sejam rastreados e comparados com o Cenário 2.

Essa abordagem possibilita o comparativo de receitas e custos relacionados a colheita e estradas obtidos em cada cenários. No entanto, dado que a não aplicação de custos de estrada no Cenário 1 equivaleria a utilização do valor $\mathrm{R} \$ 0,00 \mathrm{Km}^{-1}$ em cada segmento de estrada, impactaria na perda da rastreabilidade dos segmentos, uma vez que os segmentos selecionados resultariam em valor zero. Portanto, tendo em vista contornar esse problema, foi aplicado o custo de $\mathrm{R} \$ 0,01 \mathrm{Km}^{-1}$ para todos os tipos de estradas presentes no grafo do Cenário 1 , garantindo que o custo não impactasse no resultado do modelo e bem como que as variáveis de estradas não fossem zeradas.

No Cenário 2 foram aplicados custos fixos de manutenção para toda a rede de estradas não pavimentada, cuja classificação foi definida em estradas secundárias, principais e municipais. As estradas secundárias são estradas estreitas que não possuem cascalho e possuem um custo de manutenção por quilômetro $480 \%$ menor que as estradas principais. As estradas principais correspondem ao maior custo de manutenção por quilômetro, uma vez que são estradas cascalhadas e demandam mais investimento. Já, as estradas municipais são estradas cascalhadas, pertencentes ao município, e conforme são utilizadas, o proprietário assume parte dos custos de manutenção envolvidos nessa operação, que correspondem a um quarto do valor das estradas principais. Em contrapartida, para os segmentos de estradas do tipo asfaltada foi aplicado um valor simbólico de $\mathrm{R} \$ 0,01 \mathrm{Km}^{-1}$, haja vista não zerar o valor dos segmentos de estradas selecionadas pelo modelo matemático. Nessa base de dados, a construção de novas estradas não foi uma opção, e apenas a manutenção das estradas existentes foi considerada.

As estimativas de receita e custos foram obtidas com a empresa e calculados com base em uma taxa anual de desconto de $7,5 \%$. A receita da colheita foi estimada a partir do volume de venda de madeira de processo e serraria, e descontados os custos operacionais de colheita. A partir da diferença entre a receita e o custo operacional de colheita, definiu-se o parâmetro $l_{i j}$. 
As premissas estabelecidas competem ao modelo atender aos seguintes pré-requisitos: produção mínima anual de madeira de processo equivalente a $27,8 \%$ da sua capacidade produtiva; e, atender a idade mínima e máxima de corte equivalente a 13 e 18 anos, respectivamente.

\section{RESULTADOS E DISCUSSÃO}

Problemas de planejamento tático florestal que integram o agendamento da colheita florestal e o planejamento das estradas são considerados complexos. Isso porque, esse tipo de problema tende lidar com um grande número de restrições e variáveis de decisão-na formulação dos modelos PLIM, impossibilitando, em muitos casos, uma solução inteira ótima dentro do tempo limite de processamento definido (SILVA et al., 2016). No entanto, apesar dos cenários testados no presente estudo terem resultado em 27.090 variáveis contínuas, 33.845 variáveis inteiras (binárias) e 27.910 restrições, a abordagem PLIM pode resolver com eficiência as otimizações, os quais resultaram em uma solução inteira ótima em menos de um minuto (Tabela 1 ).

Tabela 1. Comparativo de retorno corrente entre cenários que não integraram e que consideraram as estradas florestais em um planejamento de colheita florestal.

\begin{tabular}{ccccc}
\hline Cenários & $\begin{array}{c}\text { Receita } \\
\text { colheita } \\
\text { florestal* }\end{array}$ & $\begin{array}{c}\text { Custos } \\
\text { manutenção de } \\
\text { estradas* }\end{array}$ & Total* & $\begin{array}{c}\text { Tempo } \\
\text { (s)** }\end{array}$ \\
\hline 1 & 103,7 & $-3,7$ & $\mathbf{1 0 0 , 0}$ & 1,07 \\
2 & 103,6 & $-3,0$ & $\mathbf{1 0 0 , 6}$ & 9,89 \\
\hline
\end{tabular}

*Por questões de confidencialidade dos dados, os resultados foram expressos em termos relativos considerando por base o valor total do Cenário 1 cujo valor foi arbitrado em 100, e nos demais, foi mantida a proporcionalidade dos valores reais.

**Tempo de processamento computacional.

A Tabela 1 também apresentou que o Cenário 1, cujos custos de estradas não foram considerados no processo de otimização do modelo, obteve maior receita na operação de colheita florestal que em relação ao Cenário 2 (Tabela 1). Essa abordagem permite o modelo matemático ficar menos restritivo que o Cenário 2 e mais relaxado quanto a maximização da receita líquida do planejamento. Em contrapartida, quando considerado o rastreio dos segmentos de estrada selecionados no Cenário 1 e multiplicados pelos custos reais de manutenção de estradas, conforme atribuído no Cenário 2, o Cenário 1 resultou em menor lucratividade.

O maior custo de manutenção de estradas obtido no Cenário 1 está atrelado a maior distância de estradas agendadas no planejamento, que resultou em $21 \%$ de quilômetros a mais que em relação ao Cenário 2 (Tabela 2 e Figura 2). Além disso, o Cenário 1 agendou $8 \%$ e $36 \%$ a mais de estradas secundárias e estradas principais, respectivamente, que em relação ao Cenário 2. Assim, esses tipos de estradas representam os dois maiores custos de manutenção do estudo, os quais impactam de maneira expressiva o custo total de manutenção do planejamento.

Resultados similares ao do presente trabalho foram obtidos por Silva et al. (2016), que testaram cenários de planejamento integrado em uma base de dados real visando minimizar os custos totais. Nesse trabalho, os autores destacaram que os cenários que não integraram as estradas no modelo matemático resultaram em aproximadamente $60,2 \%$ quilômetros a mais de estradas agendadas, resultando em um impacto direto no aumento dos custos totais destes cenários. Além disso, Augustynczik et al. (2016) integraram custos de estradas a um planejamento florestal estratégico e obtiveram um aumento de $1,1 \%$ de lucratividade, quando comparado a um cenário sem investimento rodoviário.

Tabela 2. Classificação e discriminação da distância total de estradas selecionadas em cada cenário testado.

\begin{tabular}{ccc}
\hline Tipos de & Distância total de estradas selecionadas $(\mathbf{k m})$ \\
\cline { 2 - 3 } estradas & Cenário 1 & Cenário 2 \\
\hline Secundária & 212,86 & 196,4 \\
\hline Principal & 74,59 & 54,78 \\
Municipal & 302,77 & 254,37 \\
Asfalto & 98,52 & 64,86 \\
\hline Total & $\mathbf{6 8 8 , 7 4}$ & $\mathbf{5 7 0 , 4 1}$ \\
\hline
\end{tabular}

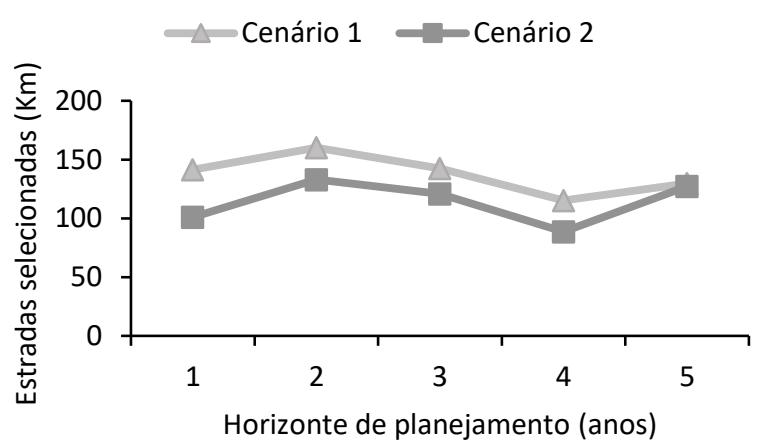

Figura 2. Distância total de estradas agendadas em cada período de planejamento dos cenários testados.

Além da importância econômica obtida em planejamentos que integram o agendamento da colheita com o planejamento de estradas, há também benefícios ambientais, uma vez que a tendência do modelo é agrupar as áreas de corte, tendo em vista minimizar a manutenção de estradas. A redução da fragmentação da floresta auxilia 
na manutenção da umidade interna das florestas (ROSS et al., 2018), bem como na minimização de ruído de máquinas e do transporte de maquinário e equipe entre unidades de corte. No entanto, quando o índice de agregação é alto, restrições de limitação de corte devem ser impostas ao modelo, as quais não foram abordadas neste estudo. Conforme a Figura 3, observa-se que o Cenário 2 apresentou as áreas de corte anuais mais próximas, tendendo selecionar menos segmentos de estradas para minimizar os custos de manutenção de estradas.

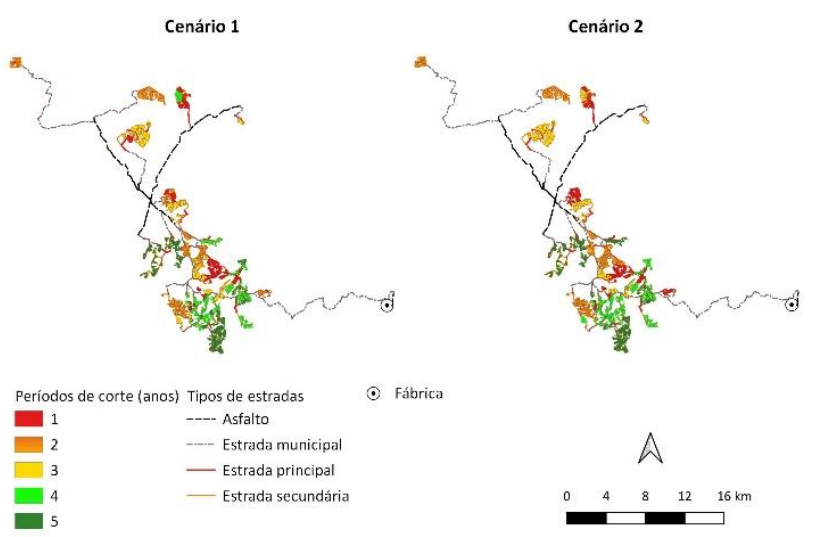

Figura 3. Comparativo da espacialização entre os cenários 1 e 2.

\section{CONCLUSÕES}

A abordagem de programação linear inteira mista pode resolver com eficiência os cenários propostos, uma vez que resultaram em uma solução inteira ótima inferior a um minuto de processamento computacional. Além disso, constatou-se que a aplicação de um modelo integrado é mais eficaz que a abordagem em duas etapas vinculadas, uma vez que foi capaz de associar os benefícios-custos gerados entre a relação colheita e estradas, garantindo maior retorno financeiro para o plano.

Considerando o baixo tempo computacional demandados pelos cenários para atingir uma solução ótima do modelo matemático, sugere-se para estudos futuros o acréscimo de restrições ambientais (ex: restrições de adjacência e de green-up delay) no modelo proposto.

\section{AGRADECIMENTOS}

Os autores agradecem à Coordenação de Aperfeiçoamento de Pessoal de Nível Superior (CAPES) pelo apoio financeiro.

\section{REFERÊNCIAS}

ALVARES, C.A. et al. Köppen's climate classification map for Brazil. Meteorologische Zeitschrift, v.22, n.6, p.711-728, 2013.

AUGUSTYNCZIK, A.L.D. et al. Aggregating forest harvesting activities in forest plantations through integer linear programming and goal programming. Journal of Forest Economics, v.24, p.72-81, 2016.

BELAVENUTTI, P. et al. Integrating strategic and tactical forestmanagement models within a multicriteria context. Forest Science, v.65, n.2, p.178-188, 2019.

BORGES, J.G. et al. Strategic management scheduling. In: The management of industrial forest plantations. Springer, 2014.

D'AMOURS, S. et al. Operations research in forestry and forest products industry. Wiley Encyclopedia of Operations Research and Management Science, p.1-18, 2010.

DIJKSTRA, E.W. A note on two problems in connexion with graphs. Numerische Mathematik, v.271, p.269-271, 1959.

GUIGNARD, M. et al. Model tightening for integrated timber harvest and transportation planning. European Journal of Operational Research, v.111, n.3, p.448-460, 1998.

KIRBY, M.W. et al. Simultaneous planning of wildland management and transportation alternatives. TIMS Studies in the Management Sciences, v.21, p.371-387, 1986.

MCDILL, M.E. An overview of forest management planning and information management. In: The management of industrial forest plantations. Springer, 2014.

MOBTAKER, A. et al. Integrated forest harvest planning and roadbuilding model with consideration of economies of scale. Canadian Journal of Forest Research, v.50, p.989-1001, 2020.

NADERIALIZADEH, N. et al. Solving the integrated forest harvest scheduling model using metaheuristic algorithms. Operational Research, v.20, p.2283-2306, 2020.

RÖNNQVIST, M. et al. Operations research challenges in forestry: 33 open problems. Annals of Operations Research, v.232, n.1, p.11-40, 2015.

ROSS, K.L. et al. Forest harvest scheduling with endogenous road costs. Interfaces, v.48, n.3, p.1-11, 2018.

SILVA, P.H.B.M. et al. Plano de agendamento da colheita florestal integrado à rede de estradas. Cerne, v.22, n.1, p.69-76, 2016.

WEINTRAUB, A.; NAVON, D. A forest management planning model integrating silvicultural and transportation activities. Management Science, v.22, n.12, p.1299-1309, 1976.

Recebido em 03-09-2021 Aceito em 10-11-2021 